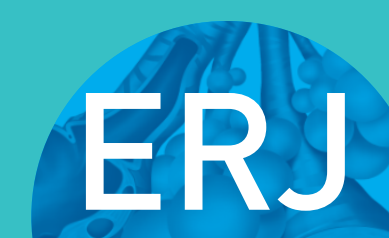

open research

\title{
Thoracic ultrasound for malignant pleural effusion: a systematic review and meta-analysis
}

\author{
Akihiro Shiroshita $\mathbb{1}^{1,2}$, Sayumi Nozaki ${ }^{3}$, Yu Tanaka², Yan Luo ${ }^{4}$ and \\ Yuki Kataoka ${ }^{5,6}$
}

Affiliations: ${ }^{1}$ Dept of Respiratory Medicine, Ichinomiyanishi Hospital, Ichinomiya, Japan. ${ }^{2}$ Dept of Pulmonology, Kameda Medical Center, Kamogawa, Japan. ${ }^{3}$ Post Graduate Education Center, Kameda Medical Center, Kamogawa, Japan. "Dept of Health Promotion and Human Behavior, Graduate School of Medicine, Kyoto University, Kyoto Japan. ${ }^{5}$ Dept of Respiratory Medicine, Hyogo Prefectural Amagasaki General Medical Center, Amagasaki, Japan. ${ }^{6}$ Dept of Hospital Care Research Unit, Hyogo Prefectural Amagasaki General Medical Center, Amagasaki, Japan.

Correspondence: Akihiro Shiroshita, Dept of Respiratory Medicine, Ichinomiyanishi Hospital, 1 Kaimeihira, 494-0001, Ichinomiya, Japan. E-mail: akihirokun8agmail.com

ABSTRACT This systematic review aimed to evaluate the diagnostic accuracy of thoracic ultrasound in malignant pleural effusion.

Articles published until December 2019 in MEDLINE, Embase, the Cochrane Central Register of Controlled Trials and the International Clinical Trials Registry Platform were screened by two authors independently to extract data and evaluate the risks of bias and applicability using the modified Quality Assessment of Diagnostic Accuracy Studies-2 tool. We described the forest plots of each thoracic ultrasound finding. We estimated the pooled sensitivity and specificity of pleural nodularity using the bivariate random-effects model.

We included seven articles and found that each thoracic ultrasound finding had low sensitivity. The pooled specificity of pleural nodularity was $96.9 \%$ (95\% CI $93.2 \%-98.6 \%$ ).

In conclusion, thoracic ultrasound is not useful in ruling out malignant pleural effusion. Physicians can proceed rigorously to repeat thoracentesis or other invasive procedures when pleural nodularity is detected.

@ERSpublications

This systematic review shows that thoracic ultrasound cannot rule out malignant pleural effusion. Pleural nodularity could be a ruling-in test for performing repeated thoracentesis or other invasive procedures when malignant pleural effusion is suspected. https://bit.ly/3iuM5z7

Cite this article as: Shiroshita A, Nozaki S, Tanaka Y, et al. Thoracic ultrasound for malignant pleural effusion: a systematic review and meta-analysis. ERJ Open Res 2020; 6: 00464-2020 [https:// doi.org/10.1183/23120541.00464-2020].

This article has supplementary material available from openres.ersjournals.com

This study is registered at the International Prospective Register of Systematic Reviews (CRD42020162846).

Received: 4 July 2020 | Accepted after revision: 2 Sept 2020

Copyright $\odot$ ERS 2020. This article is open access and distributed under the terms of the Creative Commons Attribution Non-Commercial Licence 4.0. 


\section{Introduction}

Malignant pleural effusion (MPE) is a common malignancy complication [1]. As patients with MPE usually have poor prognoses, a prompt diagnosis is crucial to allow patients to start optimal treatment as early as possible [2]. A diagnostic thoracentesis is the first step in detecting MPE; however, the initial cytological evaluation only has a sensitivity of approximately 50-70\% [1]. If the initial thoracentesis fails to provide a definite diagnosis, pulmonologists or radiologists have to either repeat it or choose another invasive procedure, such as image-guided biopsy or thoracoscopy [3].

Ultrasound is a noninvasive and inexpensive tool; therefore, it is increasingly used by physicians [4]. Its other advantages include lack of radiation exposure and easy personal training because of easy bedside accessibility [5].

The international guidelines recommended ultrasound guidance when performing diagnostic thoracentesis to reduce the risk of complications [6, 7]. Many recent, studies have explored the utility of morphological findings of transthoracic ultrasound (TUS) as a tool for detecting MPE [8-14]. However, these studies had a small sample size and were conducted at a single centre; hence, the diagnostic accuracy of TUS remains unclear. Our systematic review aimed to evaluate the diagnostic accuracy of TUS both as a triage test and an add-on test in patients with suspected MPE.

\section{Material and methods}

The protocol of this systematic review was registered in the International Prospective Register of Systematic Reviews (CRD42020162846). Our systematic review is based on the Preferred Reporting Items for Systematic Review and Meta-Analysis for Diagnostic Test Accuracy (supplementary table S1). Informed consent from study participants was waived because of the study design. We performed a comprehensive search of MEDLINE, Embase, the Cochrane Library and the International Clinical Trials Registry Platform for publications until December 25, 2019, without any limitations on the language or publication status. Our search terms were based on TUS (index test), MPE (target condition) and specific morphological findings of TUS, including pleural thickening, hepatic metastases, pleural nodules, diaphragmatic thickening, diaphragmatic nodules, solitary pulmonary lesions and swirling (supplementary table S2). We reviewed all the reference lists of the included articles and searched the citations with Web of Science to search for additional relevant articles.

Two authors (AS and SN) independently screened the title and abstracts of the listed articles and subsequently reviewed the complete text of potential articles. The inclusion criteria were prospective or retrospective observational studies, case-control studies or case series that assessed the sensitivity and specificity of morphological findings of TUS for MPE. We carefully confirmed that all included studies reported obtaining informed consent from each study participant and protocol approval by an ethics committee or institutional review board. The exclusion criteria were: 1) case reports, review articles, or articles that used animal models; and 2) studies that used ultrasound on lesions other than those in the lung (e.g. abdominal ultrasound). We extracted the following details of the included articles: study design, participants, index tests, reference standards and diagnostic accuracy.

AS and SN independently evaluated the risk of bias and concerns of applicability of the included articles using the modified Quality Assessment of Diagnostic Accuracy Studies-2 (QUADAS-2) tool [14]. During the entire review process, disagreements between the two authors were resolved through discussions and consultations with another pulmonologist, YT. Forest plots were created to illustrate the diagnostic accuracy of each index test in each study. Generally, there are four types of TUS findings in patients with MPE: 1) gross macroscopic findings, including echogenicity and swirling sign; 2) pleural thickness, in which different thresholds may be used; 3) nodularity of parietal or visceral pleura, or the diaphragm; and 4) other findings, such as parenchymal lesions and hepatic metastases. Although we planned to estimate the pooled sensitivity and specificity for each type of finding using a bivariate random-effects model, we expected that the sensitivity and specificity of each TUS finding might vary widely. Therefore, we visually checked the heterogeneity for each finding on the forest plots, moreover, we only calculated the pooled sensitivity and specificity for findings lacking apparent heterogeneity. In addition, we described the hierarchical summary receiver operating characteristic (HSROC) curve for these findings. The overall quality of evidence of pleural nodularity was assessed using the Grading of Recommendations, Assessment, Development and Evaluation (GRADE) approach [15].

For statistical analysis, we used R 3.6.0 (R Foundation for Statistical Computing, Vienna, Austria) to generate forest plots; STATA 15 (STATA Corp., College Station, TX, USA) to calculate the pooled sensitivity and specificity; and RevMan v.5.3 (The Nordic Cochrane Centre, The Cochrane Collaboration, Copenhagen, Denmark) to summarise the risk of bias and applicability and to create the HSROC curve. 


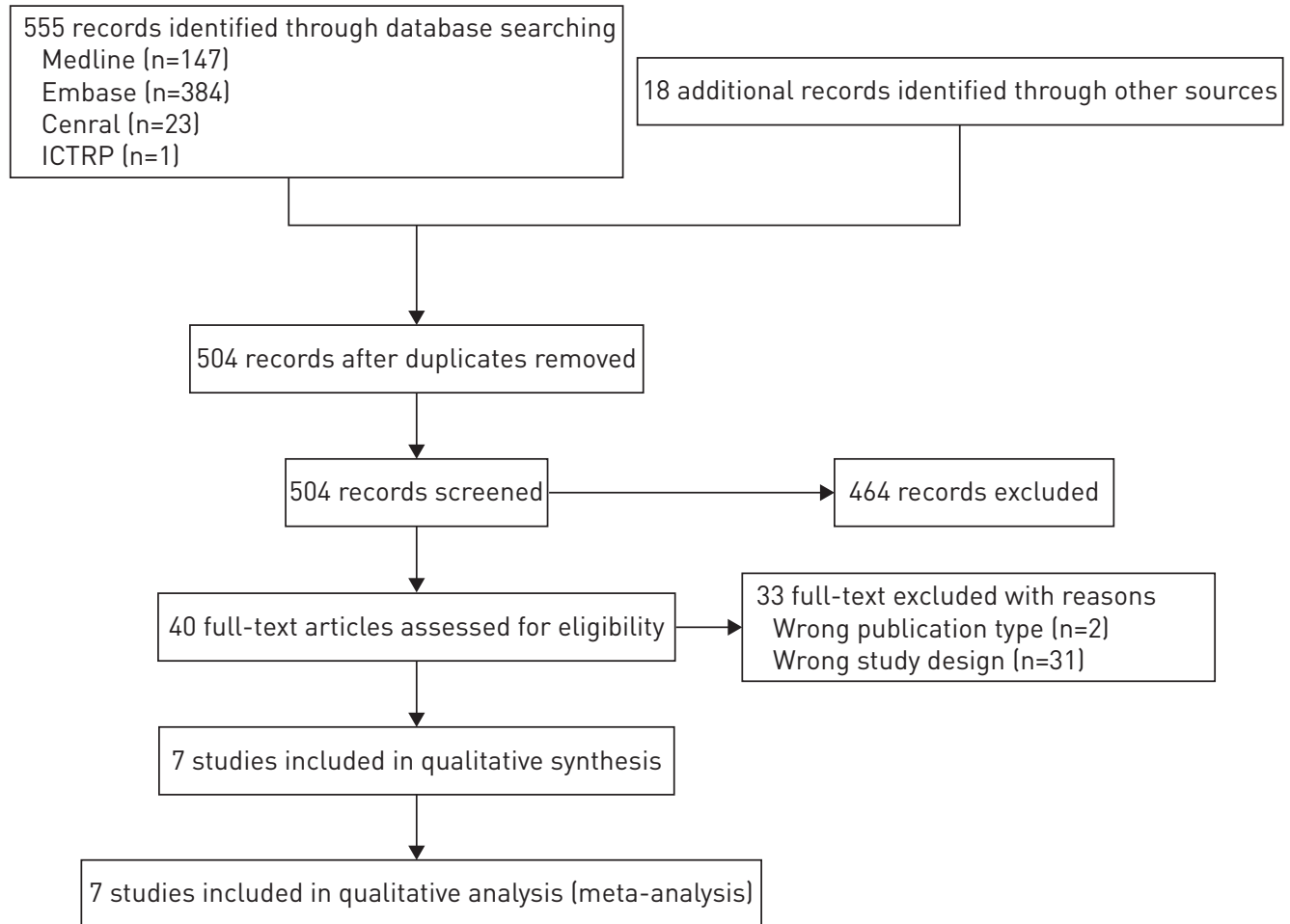

FIGURE 1 Preferred Reporting Items for Systematic Review and Meta-Analysis flow diagram. ICTRP: International Clinical Trials Registry Platform.

\section{Results}

Figure 1 illustrates the study selection process. After removing duplicates, we screened 504 articles and included seven studies after applying the exclusion criteria $[8-13,16]$. Table 1 summarises the characteristics of the included articles. The included articles assessed 840 patients. All the included articles were prospective studies. Experienced radiologists or pulmonologists performed TUS at a university hospital or tertiary care centre (table 2). Regardless of the follow-up periods, pathological results, including cytology or other biopsy results, were used as a reference standard.

Supplementary figures S1 and S2 summarise the quality of each study using the modified QUADAS-2 tool. Regarding the risk of bias, the reference standard domain was labelled as unclear because we could

\section{TABLE 1 Characteristics of the included studies}

\begin{tabular}{|c|c|c|c|c|c|c|c|c|}
\hline $\begin{array}{l}\text { First author } \\
\text { [ref.] }\end{array}$ & Year & Participants n & Mean age (so) & Male \% & $\begin{array}{l}\text { Prevalence } \\
\text { of MPE \% }\end{array}$ & Study design & Country & Study setting \\
\hline $\mathrm{Y}_{\text {ANG }}[16]$ & 1992 & 320 & $54(16)$ & 59 & 35 & $\begin{array}{c}\text { Prospective } \\
\text { cohort }\end{array}$ & China & University hospital \\
\hline Lomas [11] & 1993 & 86 & 58 & 64 & 34 & $\begin{array}{c}\text { Prospective } \\
\text { cohort }\end{array}$ & United Kingdom & University hospital \\
\hline MARCun [12] & 2009 & 40 & 58 & 67 & 23 & $\begin{array}{c}\text { Prospective } \\
\text { cohort }\end{array}$ & Slovenia & University hospital \\
\hline Bugalho [9] & 2014 & 133 & $67(16)$ & 46 & 50 & $\begin{array}{c}\text { Prospective } \\
\text { cohort }\end{array}$ & Portugal & University hospital \\
\hline Asciak [8] & 2018 & 140 & Not described & Not described & 45 & $\begin{array}{c}\text { Prospective } \\
\text { cohort }\end{array}$ & United Kingdom & University hospital \\
\hline FaHeEm [10] & 2019 & 69 & $50(16)$ & 42 & 33 & $\begin{array}{c}\text { Prospective } \\
\text { cohort }\end{array}$ & Egypt & University hospital \\
\hline
\end{tabular}


TABLE 2 Detailed information about thoracic ultrasound

\begin{tabular}{|c|c|c|c|c|}
\hline $\begin{array}{l}\text { First author } \\
\text { [ref.] }\end{array}$ & Machine & Probe & Operators and interpreters & Position \\
\hline YANG [16] & $\begin{array}{l}\text { Aloka SSD 630, SSD } 650, \\
\text { Aloka, Tokyo, Japan; } \\
\text { Toshiba 100A, } \\
\text { Toshiba, Tokyo, Japan }\end{array}$ & $\begin{array}{l}\text { 3.5-, } 5.0-\text { and } 7.5-\mathrm{MHz} \text { linear and } \\
\text { convex transducers }\end{array}$ & $\begin{array}{l}\text { One of three sonographers performed the } \\
\text { ultrasound. The images were recorded } \\
\text { on Polaroid film (Polaroid, Cambridge, } \\
\text { MA, USA) and were interpreted by the } \\
\text { other two sonographers }\end{array}$ & $\begin{array}{l}\text { Sitting or } \\
\text { supine }\end{array}$ \\
\hline MARCun [12] & ATL HDI 5000CV & $\begin{array}{l}\text { Phase array } \mathrm{P} 4-2 \text { convex } \\
\text { transducer }\end{array}$ & $\begin{array}{l}\text { An experienced pulmonologist conducted } \\
\text { ultrasound and the images were stored } \\
\text { on hard disc for further evaluation }\end{array}$ & $\begin{array}{l}\text { Sitting or } \\
\text { supine }\end{array}$ \\
\hline QuREshi [13] & $\begin{array}{l}\text { A single Esaote Technos } \\
\text { MPX } 25\end{array}$ & $\begin{array}{l}\text { A 3-5-MHz curvilinear probe } \pm \\
8-15 \mathrm{MHz} \text { linear probe (to } \\
\text { visualise the pleura and chest } \\
\text { wall in greater detail) }\end{array}$ & $\begin{array}{l}\text { An experienced radiologist performed the } \\
\text { ultrasound and the images were stored } \\
\text { as anonymised data. Consultant } \\
\text { radiologists separately reviewed them }\end{array}$ & $\begin{array}{l}\text { Upright or } \\
\text { lateral } \\
\text { decubitus }\end{array}$ \\
\hline Asciak [8] & Hitachi Avius & The abdomen pre-set & $\begin{array}{l}\text { The ultrasound reporters held a } \\
\text { minimum of Royal College of } \\
\text { Radiologists level } 1 \text { accreditation, and } \\
\text { at least two sonographers performed } \\
\text { the pre-procedure ultrasound scan and } \\
\text { agreed on the described echogenic } \\
\text { qualities of the fluid }\end{array}$ & Not described \\
\hline FAHEEM $[10]$ & GE logiq P6 pro & $\begin{array}{l}\text { Convex array }(3-5 \mathrm{MHz}) \text { and linear } \\
\text { array }(4-11 \mathrm{MHz})\end{array}$ & Not described & $\begin{array}{l}\text { Supine, prone, } \\
\text { lateral or } \\
\text { sitting }\end{array}$ \\
\hline
\end{tabular}

not ascertain whether the pathologists were blinded in all the articles. In one article by FAHEEM [10], the risk of bias in the index test domain was high because the ultrasound operators were unblinded.

Figures 2 and 3 illustrate the forest plots of sensitivity and specificity according to each index test. The gross macroscopic findings were assessed and echogenicity, a specific sign of MPE, demonstrated a wide range of sensitivity and specificity. Parietal thickness was evaluated using different cut-off values, $3 \mathrm{~mm}$ versus $10 \mathrm{~mm}$. Pleural thickness assessment demonstrated low sensitivity and varying specificity. Although only two studies used a cut-off value of $10 \mathrm{~mm}$, they revealed high specificity. We did not calculate the pooled sensitivity and specificity of either echogenicity or parietal pleural thickness given the heterogeneity of the results. Nodularity was assessed in the parietal pleura, visceral pleura or diaphragm. The pooled sensitivity and specificity of nodularity was 42.5\% (95\% CI $25.3 \%-61.6 \%)$ and $96.9 \%$ (95\% CI 93.2\%-98.6\%), respectively, using the bivariate random effect model. Additionally, the HSROC curve revealed high specificity (supplemental figure S3). Finally, we evaluated the overall quality of evidence of pleural nodularity using the GRADE approach, which showed a moderate certainty of evidence (table 3 ).

\section{Discussion}

This systematic review revealed that each macroscopic finding on TUS demonstrated low sensitivity and a wide range of specificity. It demonstrated high specificity and moderate overall quality of evidence for pleural nodularity, including the parietal and somatic pleural nodules and diaphragmatic nodules.

As pleural nodularity has a high specificity and positive predictive value, it can be used as an add-on test for ruling-in MPE. Cytopathological evaluations, such as cytology or cell blocks, can contribute to a definite diagnosis; however, one-time thoracentesis demonstrated low sensitivity [7]. In case chest physicians or radiologists detect pleural nodules during TUS, the pre-test probability of MPE may be increased and repeat thoracentesis or other invasive procedures can be justified. However, physicians 


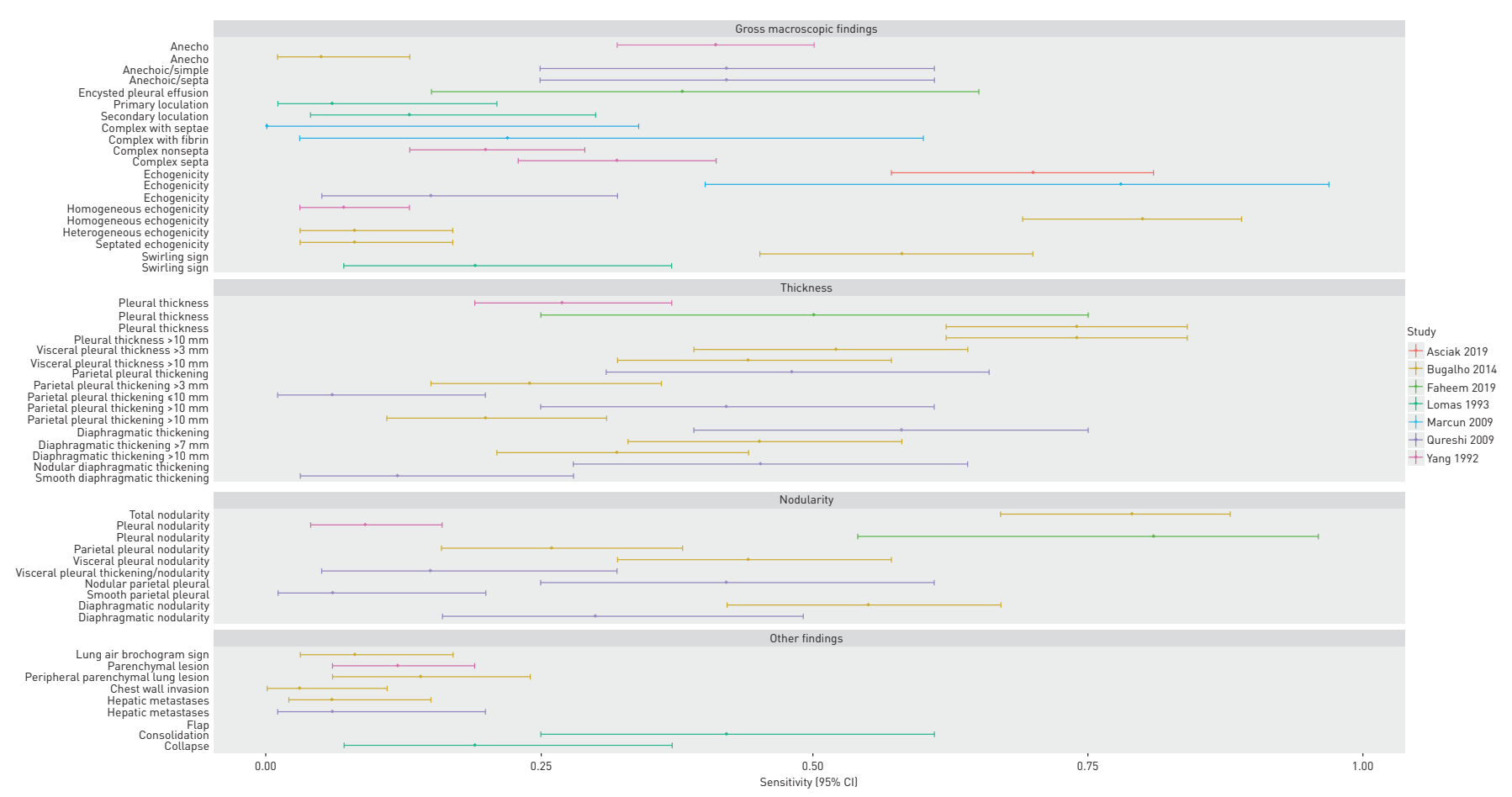

FIGURE 2 Forest plot of the sensitivity for each ultrasound finding in malignant pleural effusion.

should keep in mind that biopsy in pleural or other sites could guide the treatment more precisely based on specific histological subtypes and molecular patterns.

Contrary to pleural nodularity, other index tests demonstrated low specificity and therefore cannot be used as add-on tests. In the current systematic review, we evaluated the diagnostic accuracy of each morphological finding on TUS. Future studies should combine assessments of each of these findings [13].

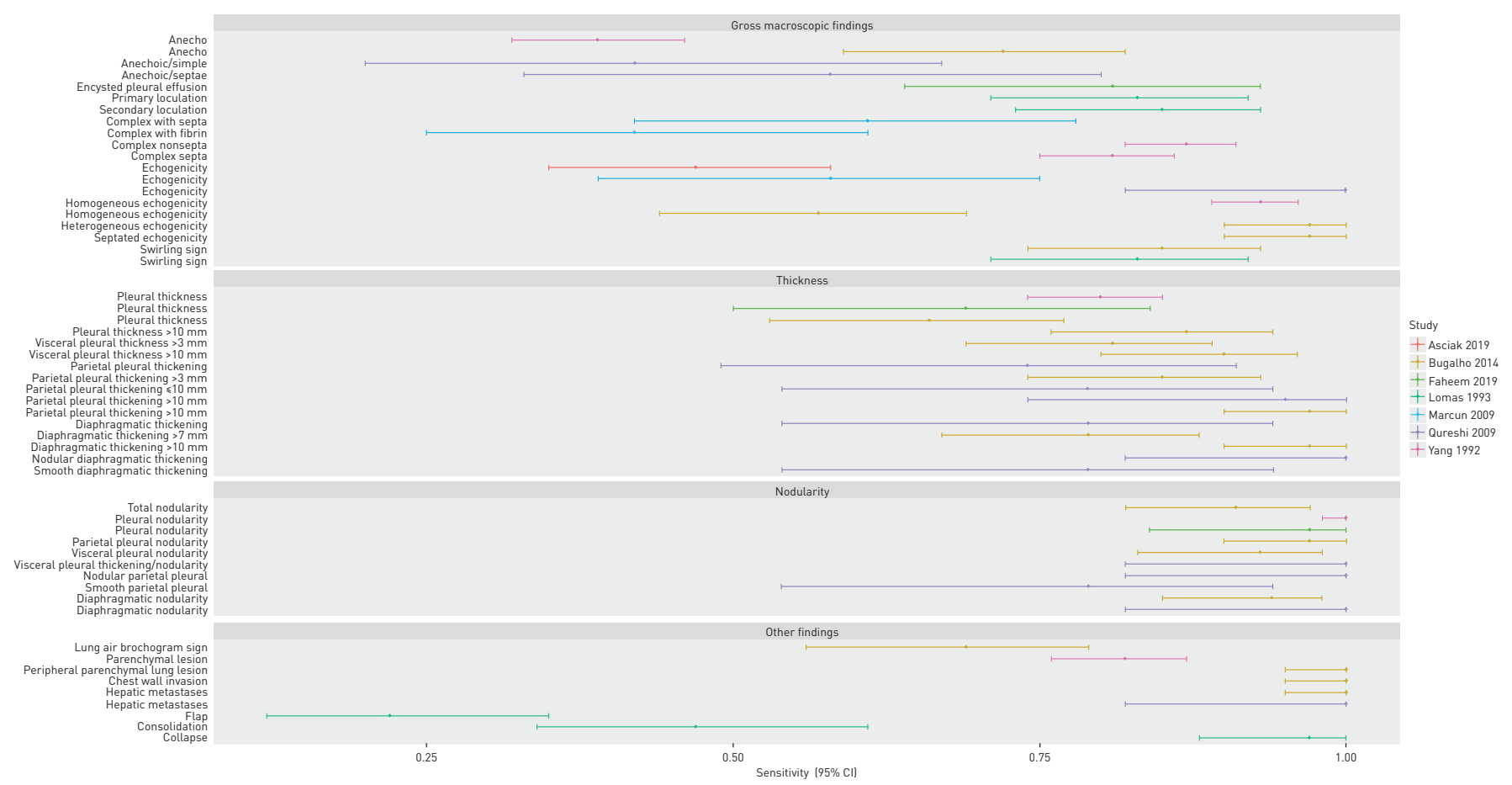

FIGURE 3 Forest plot of the specificity of each ultrasound finding in malignant pleural effusion. 
TABLE 3 Findings with the pleural nodularity via thoracic ultrasound using the Grading of Recommendations, Assessment, Development and Evaluation approach

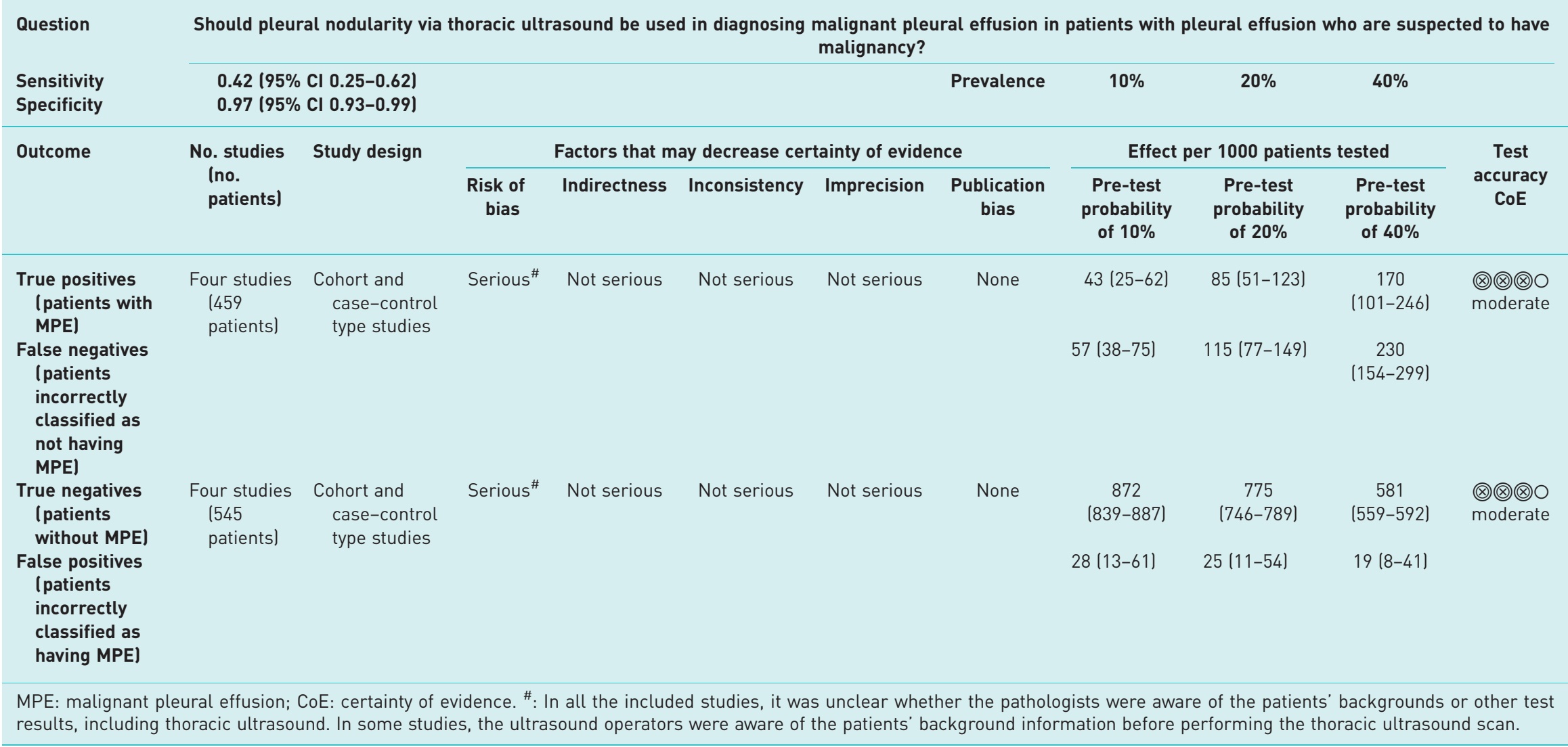


QURESH et al. [13] calculated the sensitivity and specificity of combining nodularity, pleural thickening $>1 \mathrm{~cm}$ and hepatic metastasis. They found that this combination demonstrated extremely high specificity compared with contrast-enhanced computed tomography (sensitivity $73 \%$, specificity $100 \%$ ). We could not identify any other articles that reported the overall diagnostic yield. Currently, physicians cannot use any other single morphological pattern except pleural nodularity for ruling-in MPE.

TUS cannot be used as a triage test for ruling out MPE among patients with pleural effusion who are suspected to have malignancy. The result is plausible because thoracentesis is a relatively easy and safe procedure; therefore, it only has a few contraindications, such as the presence of small pleural effusion or inability to maintain the position [17]. When MPE is suspected, it is reasonable to proceed to histopathological tests, such as thoracentesis or thoracoscopy.

This systematic review has several limitations. First, in each article, experienced radiologists or pulmonologists performed TUS in university hospitals. TUS is operator-dependent and a relatively new module. Physicians and ultrasound practitioners require further education and experience to popularise the use of TUS. There is a need for future studies in primary or secondary care settings. Second, five of the seven studies were conducted in Europe, which is not an endemic region for tuberculosis. Pleural tuberculosis, among the most common forms of extrapulmonary tuberculosis, can be visualised as pleural nodules on computed tomography [18]. There are concerns regarding the applicability of pleural nodularity in patients in tuberculosis endemic areas. Third, we could not assess publication bias and heterogeneity using statistical methods. Currently there is no valid method to test for publication bias; further, the methodology of meta-analyses for diagnostic accuracy comprises a substantial risk of bias.

In conclusion, the morphological findings of TUS were not useful as a ruling-out test. Nevertheless, pleural nodularity on ultrasound could motivate us to proceed with repeat thoracentesis or other invasive procedures when MPE is suspected.

Conflict of interest: None declared.

\section{References}

$1 \quad$ Bennett R, Maskell N. Management of malignant pleural effusions. Curr Opin Pulm Med 2005; 11: 296-300.

2 Postmus PE, Brambilla E, Chansky K, et al. The IASLC Lung Cancer Staging Project: proposals for revision of the $\mathrm{M}$ descriptors in the forthcoming (seventh) edition of the TNM classification of lung cancer. J Thorac Oncol 2007; 2: 686-693.

3 Rivera MP, Mehta AC, Wahidi MM. Establishing the diagnosis of lung cancer: diagnosis and management of lung cancer, 3rd ed: American College of Chest Physicians evidence-based clinical practice guidelines. Chest 2013; 143: e142S-e165S.

4 Koegelenberg CFN, von Groote-Bidlingmaier F, Bolliger CT. Transthoracic ultrasonography for the respiratory physician. Respiration 2012; 84: 337-350.

5 Pietersen PI, Madsen KR, Graumann O, et al. Lung ultrasound training: a systematic review of published literature in clinical lung ultrasound training. Crit Ultrasound J 2018; 10: 23.

6 Gordon CE, Feller-Kopman D, Balk EM, et al. Pneumothorax following thoracentesis: a systematic review and meta-analysis. Arch Intern Med 2010; 170: 332-339.

7 Hooper C, Lee YCG, Maskell N, et al. Investigation of a unilateral pleural effusion in adults: British Thoracic Society Pleural Disease Guideline 2010. Thorax 2010; 65: Suppl. 2, ii4-i17.

8 Asciak R, Hassan M, Mercer RM, et al. Prospective analysis of the predictive value of sonographic pleural fluid echogenicity for the diagnosis of exudative effusion. Respiration 2019; 97: 451-456.

9 Bugalho A, Ferreira D, Dias SS, et al. The diagnostic value of transthoracic ultrasonographic features in predicting malignancy in undiagnosed pleural effusions: a prospective observational study. Respiration 2014; 87: 270-278.

10 Faheem MH. Is transthoracic ultrasound (TUS) a reliable predictor of the nature of pleural and peripheral pulmonary lesions? Correlation with cyto-histological findings. Egypt J Radiol Nucl Med 2019; 50: 4.

11 Lomas DJ, Padley SG, Flower CD. The sonographic appearances of pleural fluid. Br J Rad 1993; 66: 619-624.

12 Marcun R, Sustic A. Sonographic evaluation of unexplained pleural exudate: a prospective case series. Wien Klin Wochenschr 2009; 121: 334-338.

13 Qureshi NR, Rahman NM, Gleeson FV. Thoracic ultrasound in the diagnosis of malignant pleural effusion. Thorax 2009; 64: 139-143.

14 Whiting PF, Rutjes AWS, Westwood ME, et al. QUADAS-2: a revised tool for the quality assessment of diagnostic accuracy studies. Ann Intern Med 2011; 155: 529-536.

15 Balshem H, Helfand M, Schünemann HJ, et al. GRADE guidelines: 3. Rating the quality of evidence. J Clin Epidemiol 2011; 64: 401-406.

16 Yang PC, Luh KT, Chang DB, et al. Value of sonography in determining the nature of pleural effusion: analysis of 320 cases. Am J Roentgenol 1992; 159: 29-33.

17 Puchalski JT, Argento AC, Murphy TE, et al. The safety of thoracentesis in patients with uncorrected bleeding risk. Ann Am Thorac Soc 2013; 10: 336-341.

18 Udwadia ZF, Sen T. Pleural tuberculosis: an update. Curr Opin Pulm Med 2010; 16: 399-406. 